\title{
Study of Per se Performance of Parents and Hybrids for Yield and Quality of Bittergourd (Momordica charantia L.)
}

\author{
Amrita Kumari ${ }^{1}$, Sangeeta Shree ${ }^{1 *}$, Randhir Kumar ${ }^{1}$, M. Haque ${ }^{3}$, \\ Chandan Kishor ${ }^{2}$ and V.K. Singh ${ }^{1}$ \\ ${ }^{1}$ Department of Horticulture (Vegetables and Floriculture), Bihar Agricultural University, \\ Sabour, 813210, India \\ ${ }^{2}$ Department of Plant Breeding and Genetic, Bihar Agricultural University, \\ Sabour, 813210, India \\ ${ }^{3}$ Department of Agronomy, Bihar Agricultural University, Sabour, 813210, India
}

*Corresponding author

A B S T R A C T

The present experiment was formulated on vegetable research farm, Bihar Agricultural University, Sabour to study the per se performance of 21

Keywords

Bitter gourd (Momordica charantia L.), ANOVA

Article Info

Accepted:

15 June 2019

Available Online:

10 July 2019 hybrids with seven dissimilar parents. The ANOVA for experimental design expressed that the treatment variance were highly significant for all the quantitative and qualitative traits. Fruit yield is the ultimate character which shows the utility of a genotype in farmer's field. As per the present findings, the parents Pusa Ausadhi, followed by BRBT Local and Kathi No.1 were the most promising parental line. However, among hybrids, Pusa Ausadhi X BRBT Local followed by Kathi No.1 X BRBT Local and BRBT Local X Konkan Tara were considered as best hybrid regarding fruit yield. For quality point of view, the best promising parent were found to be BRBT Local for total soluble solid (TSS) and chlorophyll content, Konkan Tara for carotenoid content, Kathi No. 1 for total phenol content and Konkan Tara for ascorbic acid. Out of 21 hybrids, BRBT Local X Konkan Tara had highest ascorbic acid content followed by Kathi No.1 X Konkan

\section{Introduction}

Bitter gourd (Momordica charantia L.) is one of the most important tropical and subtropical cucurbitaceous vegetable crops and the popularity and demand of this crop is increasing day by day among people due to the growing awareness of the antidiabetic property and nutritive value of this crop. Tropical Asia is considered as a native home of bitter gourd. Among the cucurbitaceous vegetables bitter gourd contains highest 
nutritive value (Mini Raj et al., 1993). This crop is also value for carbohydrate, protein, vitamin $\mathrm{A}$, Vitamin $\mathrm{C}$ and minerals (Raman and Lau, 1996). The name bitter gourd itself shows its taste i.e. bitter due to bitter compound called momordicin. Bitter gourd contains an array of biologically active plant chemicals including triterpenes, proteins, steroids, alkaloids, saponins, flavonoids and acids due to which plant possesses antifungal, antibacterial, antiparasitic, antiviral, antifertility, antitumorous, hypoglycemic and anticarcinogenic properties (Beloin et al., 2005, Grover and Yadav, 2004 and Zafar, 1991).

It is a potent hypoglycemic agent due to alkaloids and insulin like peptides and a mixture of steroidal sapogenins known as charantin (Agrawal, 2004). In India, it has accumulated a wide range of variability with respect to different quantitative and qualitative traits. Bitter gourd grown in different parts of the tropics varies widely in fruit colour, seed colour, fruit size etc. (Behera et al., 2008). Thus, preferences also vary from region to region (Dey et al., 2010). For example, dark green, long $(15-40 \mathrm{~cm})$ fruited types are preferred in North India where as white and medium long $(12-20 \mathrm{~cm})$ fruits are liked in South India. However, people of East India prefer short, green fruited (Muricata) type.

These varying preferences and nutritional aspect augment the need to develop varieties with different characteristics and adaptability through both conventional and nonconventional breeding. For designing of any breeding programme, collection, characterisation and evaluation of germplasm and selection of parents from them are very essential and crucial step for crop improvement. Hence, in the present study try to understand the per se performance of parents and hybrids for yield and quality of bitter gourd.

\section{Materials and Methods}

The present investigation was conducted at the research farm, Department of Horticulture (Vegetable and Floriculture) of Bihar Agricultural College, Sabour, Bhagalpur (Bihar) during two summer season of 2016 and 2017. The experimental material for this experiment comprised of seven promising and diverse genotypes i.e., Pusa Ausadhi, Jhalri, Kathi No.1, Pusa Rasdar, BRBT Local, Konkan Tara, Solan Hara and NBGH 167 (Standard check).

They were crossed in all possible combination in diallel fashion excluding reciprocals to get 21 hybrids. All the 29 treatments (7 parents, $21 \mathrm{~F}_{1}$ hybrids with one check) were grown in randomized block design with three replications with spacing of $2.0 \mathrm{~m} \times 0.5 \mathrm{~m}$ in plot size $4.0 \mathrm{~m} \mathrm{X} 3 \mathrm{~m}$. such that twelve plants were maintained in each plot. The recommended agronomic practices were adopted for raising the better crops.

Observations were recorded on 21 economically important traits viz; node number at which first male flower appear, node number at which first female flower appear, Days taken to first male flower appearance, Days taken to first female flower appearance, days to $50 \%$ flowering, internodal length $(\mathrm{cm})$, number of primary branches per plant, vine length $(\mathrm{cm})$, number of fruits per plant, fruit weight $(\mathrm{g})$, fruit length $(\mathrm{cm})$, fruit diameter $(\mathrm{cm})$, number of seeds per fruit, fruit fly infestation $\%$, yield of marketable fruits per plant $(\mathrm{g})$, total yield $(\mathrm{q} / \mathrm{h})$, TSS $\left({ }^{0}\right.$ Brix $)$, ascorbic acid (mg/100g), and chlorophyll content (mg/100gm) carotenoid content $(\mathrm{mg} / 100 \mathrm{gm})$ and total phenol content.

Data were taken for both consecutive year 2016 and 2017 and the pooled mean data was subjected to statistical analysis as suggested by Panse and Sukathme (1957). 


\section{Results and Discussion}

The treatment variance were highly significant for all the quantitative and qualitative traits(Table 1) like node number of 1st male flower, node number of 1st female flower, Days to 1st male flower, Days to 1st female flower, days to 50 per cent flowering, internodal length, number of primary branches, number of fruits per vine, fruit length, fruit weight, fruit diameter, number of seeds per fruit, vine length, Yield, Fruit infestation, TSS, Ascorbic acid, Total chlorophyll, Carotenoid and Total phenol content in bitter gourd. This indicates that considerable amount of variability were present in the genotypes. Hence the ample scope for inclusion of promising genotypes in breeding programme for yield and attributing traits. Similar types of studies were carried out by Sundhariya and Venkatesan (2007) and Yadav et al., (2011). Higher mean values of $\mathrm{F}_{1}$ hybrids than those of parents for all the characters studied, indicated the copious scope for the improvement of these characters. Elimination of undesirable types of genotypes is essential for developing desirable traits in any crop improvement programme which can be achieved by per se performance of parents and hybrids.

On the basis of pooled value analysis of both year, perusal of Per se performance (Table 2) of the parental lines and $F_{1}$ hybrids for all the characters studied revealed a wide range of mean values which indicated that the parental lines involved in this study were genetically diverse and had good breeding value, which confirmed the predictions of analysis of variance. Flower emergence site is node, in bitter gourd and it is responsible for emergence of male and female flower. If the flowers appeared at lower number of nodes may produce large number of flower and fruits and as consequently high yield. Among the parental lines, Pusa Ausadhi $(8.83,6.66)$ and
PusaRasdar $(10.99,14.00)$ were the earliest with respect to node number of $1^{\text {st }}$ male flower and node number of $1^{\text {st }}$ female flower appears respectively. Among the hybrids, PusaAusadhi X Konkan Tara (14.00) and PusaAusadhi X Solan Hara (14.00) had lowest node for first female flower and were desirable for yield.These finding are similar with the earlier work of scientists Laxuman (2005), Islam et al., (2014) and Singh et al., (2016) in bitter gourd.Solan Hara (42.83 days) was earliest for days taken to $1^{\text {st }}$ male flowers appearance followed by Pusa Ausadhi (43.16 days). Similarly Pusa Ausadhi was earliest with respect to days taken to $1^{\text {st }}$ female flowers appearance (42.16) and days to $50 \%$ flowering (48.67days). Out of 21 hybrids, PusaAusadhi X Konkan Tara (42.67) showed minimum number of days to $1^{\text {st }}$ female flowering and the hybrid Pusa Ausadhi $\mathrm{X}$ BRBT Local (50.67 days) followed by PusaAusadhi X PusaRasdar (51.04 days) showed earliest days taken to $50 \%$ flowering. Similar results were also found byLaxuman (2005), Islam et al., (2014) and Singh et al., (2016) in bitter gourd.For internodal length, Konkan Tara $(5.06 \mathrm{~cm})$ followed by Pusa Ausadhi $(5.22 \mathrm{~cm})$ showed minimum internodal length and the hybrid PusaAusadhi $\mathrm{X}$ Konkan Tara $(4.98 \mathrm{~cm})$ had lowest internodal length followed by PusaAusadhi X Solan Hara $(5.17 \mathrm{~cm})$ which were horticulturally desirable genotypes and hybrids for more yield in terms of more flowers per node. Similar results were also found by Rani et al., (2014) and Zehra et al., (2018). The higher the vine length, the higher will be the yield and hence in the present study, among parental lines, desirable genotypes with maximum vine length were BRBT Local $(277.95 \mathrm{~cm})$ followed by Kathi No.1 $(264.27 \mathrm{~cm})$ and promising hybrids for this trait were Kathi No.1 X BRBT Local, Kathi No.1 X BRBT Local $(300.23 \mathrm{~cm})$ and Pusa Ausadhi X BRBT Local $(288.87 \mathrm{~cm})$. Similarly, the desirable parent for number of 
primary branches was BRBT Local (13.67) followed by Pusa Ausadhi (13.17) and among 21 hybrids Pusa Ausadhi X BRBT Local (14.83) had maximum number of primary branches followed by Kathi No.1 X BRBT Local (14.66). In case of number of fruit per plant desirable parents were Konkan Tara (42.50) followed by Pusa Ausadhi (38.67) and Jhalri (38.67). The promising hybrids for this trait were PusaAusadhi X Konkan Tara (40.17) followed by PusaAusadhi $X$ Kathi No.1. (40.00). The same curve of finding in their study were observed by Rajeswari (1998), Sundaram, (2006), Rani et al., (2014) and Zehra (2018).

Table.1 Analysis of variance for design of experiment for quantitative characters in bitter

\begin{tabular}{|c|c|c|c|c|}
\hline \multirow{2}{*}{$\begin{array}{l}\text { SI. } \\
\text { No. }\end{array}$} & \multirow[t]{2}{*}{ Characters } & \multicolumn{3}{|c|}{ Mean squares } \\
\hline & & $\begin{array}{l}\text { Environments } \\
\text { (Y) d. f. =1 }\end{array}$ & $\begin{array}{l}\text { Treatments } \\
\text { (Tr)d. f. }=28\end{array}$ & $\begin{array}{c}\text { Error. (E.E) } \\
\text { d. f. = 140 }\end{array}$ \\
\hline 1. & Node no. of 1st male flower & 1.887 & $22.494 * *$ & 0.630 \\
\hline 2. & Node no. of 1 st female flower & 0.574 & $47.073 * *$ & 1.064 \\
\hline 3. & Days to 1st male flower & 0.000 & $8.657 * *$ & 8.544 \\
\hline 4. & Days to 1 st female flower & 0.0307 & $41.092 * *$ & 7.506 \\
\hline 5. & Days to $50 \%$ flowering & 0.042 & $19.158 * *$ & 7.308 \\
\hline 6. & Internodal length $(\mathrm{cm})$ & 0.366 & $4.135^{* *}$ & 0.150 \\
\hline 7. & Number of primary branch & 54.197 & $4.850^{* *}$ & 0.634 \\
\hline 8. & Number of fruit & 25.125 & $145.556 * *$ & 4.367 \\
\hline 9. & Fruit length $(\mathrm{cm})$ & 0.017 & $34.553^{* *}$ & 95.757 \\
\hline 10 & Fruit weight (g) & 1.794 & $1017.796^{* *}$ & 16.037 \\
\hline 11. & Fruit dimeter $(\mathrm{cm})$ & 0.234 & $2.290^{* *}$ & 0.056 \\
\hline 12. & Number of seed per fruit & 2.817 & $77.653 * *$ & 1.081 \\
\hline 13 & Vine length $(\mathrm{cm})$ & 822.99 & $5883.835^{* *}$ & 237.051 \\
\hline 14 & Yield (g/Vine) & 2707.53 & $433549.80^{* * *}$ & 14814.98 \\
\hline 15 & Yield (q/h) & 186.39 & $2269.626^{* *}$ & 125.29 \\
\hline 16 & Fruit infestation \% & 2.4129 & $40.711^{* *}$ & 3.72 \\
\hline 17 & TSS $\left(\right.$ Brix $\left.^{0}\right)$ & 0.063 & $2.302 * *$ & 0.048 \\
\hline 18 & Ascorbic acid (mg/100 & 39.431 & $1480.763^{* *}$ & 7.773 \\
\hline 19 & Total chlorophyll (mg/100g) & 0.120 & $0.270^{* *}$ & 0.009 \\
\hline 20 & Carotenoid (mg/100g) & 0.004 & $0.892^{* *}$ & 0.005 \\
\hline 21 & Phenol (mg CE/100g) & 4.293 & $1525.473^{* *}$ & 17.218 \\
\hline
\end{tabular}

NNMF -Node Number at Ist Male flower appears, NNFF - Node Number at Ist Female flower appears, DMF - Days to Ist Male flower appears, DFF - Days to Ist Female flower appears, D 50\% F - Days to 50\% Flowering, Intnd L (cm)- Inter Nodal Length (cm), PB - Primary Branches per plant, NFr - Fruits per plant, Fr L $(\mathrm{cm})$ - Fruit Length $(\mathrm{cm})$, FrWt (g) -Fruit Weight (g), Fr D (cm) -Fruit Diameter NSFr - Seeds/ Fruit, VnLen (cm)- Vine Length $(\mathrm{cm})$, Yld/Vine (g)- Yield/Vine (g), Yld (q/h)- Yield/ha, TSS- Total Soluble Solids (TSS), Tot chlor.-Total chlorophyll, carot.-Carotenoids, Tot Phe.-Total Phenol, Ascr.acd-Ascorbic Acid (mg/100g.) 
Table.2 Mean Performance of parents and hybrids for yield and quality traits in Bitter gourd

\begin{tabular}{|c|c|c|c|c|c|c|c|c|c|c|c|c|c|c|}
\hline S. No. & Characters & NNMF & NNFF & DMF & DFF & D 50\% F & $\begin{array}{c}\text { Intnd L } \\
(\mathbf{c m})\end{array}$ & PB & NFr & $\begin{array}{l}\text { Fr L } \\
(\mathrm{cm})\end{array}$ & $\begin{array}{c}\text { FrWt } \\
\text { (g) }\end{array}$ & $\begin{array}{l}\text { Fr D } \\
\text { (cm) }\end{array}$ & NSFr & $\begin{array}{c}\text { VnLen } \\
\text { (cm) }\end{array}$ \\
\hline 1 & Pusa Ausadhi & 8.83 & 6.66 & 43.16 & 42.16 & 48.67 & 5.22 & 13.17 & 38.67 & 13.17 & 55.91 & 3.39 & 14.33 & 191.70 \\
\hline 2 & Jhalri & 15.66 & 18.17 & 44.17 & 48.17 & 52.99 & 7.10 & 9.99 & 38.67 & 14.19 & 43.96 & 3.17 & 15.49 & 252.22 \\
\hline 3 & Kathi No. 1 & 14.83 & 16.49 & 45.49 & 49.83 & 54.33 & 6.16 & 12.16 & 36.33 & 10.68 & 55.69 & 4.22 & 20.00 & 264.27 \\
\hline 4 & PusaRasdar & 10.99 & 14.00 & 43.50 & 47.49 & 55.33 & 5.39 & 10.99 & 28.50 & 10.43 & 65.10 & 4.89 & 12.67 & 207.77 \\
\hline 5 & BRBT Local & 16.33 & 19.83 & 45.17 & 49.17 & 56.33 & 6.96 & 13.67 & 26.33 & 16.41 & 82.71 & 4.72 & 19.00 & 277.95 \\
\hline 6 & Konkan Tara & 13.67 & 15.16 & 46.33 & 50.49 & 53.50 & 5.06 & 10.66 & 42.50 & 9.37 & 46.13 & 3.40 & 10.83 & 217.82 \\
\hline 7 & Solan Hara & 15.33 & 18.83 & 42.83 & 45.83 & 55.16 & 6.07 & 9.66 & 31.99 & 10.20 & 46.47 & 3.30 & 21.33 & 196.23 \\
\hline 8 & Pusa Ausadhi $\times$ Jhalri & 11.83 & 14.99 & 44.83 & 44.83 & 52.00 & 6.02 & 11.83 & 37.17 & 14.22 & 58.33 & 3.47 & 12.49 & 232.88 \\
\hline 9 & PusaAusadhi $\times$ Kathi No. 1 & 14.83 & 17.16 & 43.66 & 42.99 & 52.17 & 5.86 & 12.16 & 40.00 & 13.52 & 61.30 & 3.58 & 12.83 & 219.27 \\
\hline 10 & PusaAusadhi $\times$ PusaRasdar & 11.66 & 14.49 & 45.66 & 44.66 & 51.04 & 5.64 & 11.16 & 36.00 & 11.52 & 63.57 & 4.22 & 10.99 & 208.12 \\
\hline 11 & Pusa Ausadhi $\times$ BRBT Local & 12.49 & 14.83 & 45.17 & 47.17 & 50.67 & 5.56 & 14.83 & 25.99 & 17.92 & 91.43 & 4.84 & 16.66 & 288.87 \\
\hline 12 & PusaAusadhi $\times$ Konkan Tara & 11.99 & 14.00 & 44.49 & 42.67 & 54.16 & 4.98 & 11.66 & 40.17 & 12.46 & 55.59 & 3.35 & 11.33 & 201.99 \\
\hline 13 & PusaAusadhi $\times$ Solan Hara & 12.66 & 14.00 & 45.49 & 43.50 & 54.17 & 5.17 & 10.99 & 36.17 & 11.11 & 54.45 & 3.19 & 16.17 & 189.24 \\
\hline 14 & Jhalri $\times$ Kathi No.1 & 16.17 & 19.17 & 46.33 & 49.11 & 54.99 & 7.31 & 11.49 & 38.83 & 15.08 & 50.85 & 3.26 & 18.17 & 252.77 \\
\hline 15 & Jhalri $\times$ PusaRasdar & 14.16 & 17.16 & 44.33 & 48.50 & 53.33 & 5.76 & 9.66 & 32.66 & 15.62 & 58.26 & 3.83 & 10.83 & 263.00 \\
\hline 16 & Jhalri $\times$ BRBT Local & 15.49 & 19.67 & 44.33 & 48.49 & 52.83 & 7.98 & 11.16 & 38.33 & 16.13 & 52.50 & 3.47 & 14.83 & 270.88 \\
\hline 17 & Jhalri $\times$ Konkan Tara & 14.33 & 17.83 & 45.49 & 48.33 & 54.49 & 6.90 & 9.83 & 38.83 & 12.74 & 49.27 & 3.22 & 10.16 & 241.81 \\
\hline 18 & Jhalri $\times$ Solan Hara & 15.33 & 19.00 & 46.17 & 49.33 & 53.33 & 6.73 & 10.16 & 38.17 & 14.25 & 44.92 & 3.05 & 14.83 & 256.50 \\
\hline 19 & Kathi No. $1 \times$ PusaRasdar & 15.49 & 16.49 & 44.00 & 47.49 & 54.33 & 5.55 & 12.83 & 38.99 & 13.20 & 56.01 & 3.71 & 11.49 & 226.77 \\
\hline 20 & Kathi No. $1 \times$ BRBT Local & 16.67 & 18.50 & 45.67 & 48.83 & 55.33 & 6.68 & 14.66 & 35.17 & 15.63 & 83.13 & 4.76 & 17.16 & 300.23 \\
\hline 21 & Kathi No. $1 \times$ Konkan Tara & 15.17 & 17.49 & 45.99 & 49.83 & 55.83 & 6.12 & 12.33 & 34.49 & 11.80 & 61.26 & 3.26 & 14.00 & 221.28 \\
\hline 22 & Kathi No. $1 \times$ Solan Hara & 16.17 & 19.33 & 46.17 & 48.67 & 55.67 & 6.43 & 11.16 & 34.49 & 12.11 & 57.07 & 3.56 & 19.17 & 237.40 \\
\hline 23 & PusaRasdar $\times$ BRBT Local & 13.00 & 16.33 & 47.17 & 50.49 & 54.17 & 5.30 & 11.99 & 26.99 & 14.05 & 81.11 & 4.87 & 13.33 & 229.37 \\
\hline 24 & PusaRasdar $\times$ Konkan Tara & 11.16 & 14.33 & 47.83 & 49.49 & 55.33 & 5.59 & 10.66 & 27.33 & 10.16 & 75.08 & 4.24 & 9.83 & 223.10 \\
\hline 25 & PusaRasdar $\times$ Solan Hara & 12.83 & 16.49 & 45.49 & 48.49 & 53.83 & 6.76 & 10.66 & 30.16 & 10.81 & 64.50 & 4.21 & 12.33 & 191.43 \\
\hline 26 & BRBT Local $\times$ Konkan Tara & 15.49 & 19.17 & 47.33 & 50.83 & 55.16 & 7.18 & 13.83 & 33.16 & 17.30 & 78.28 & 4.44 & 20.67 & 281.33 \\
\hline 27 & BRBT Local $\times$ Solan Hara & 15.66 & 20.49 & 44.67 & 47.49 & 57.16 & 7.66 & 12.17 & 24.50 & 15.60 & 74.72 & 4.56 & 22.83 & 276.90 \\
\hline 28 & Konkan Tara $\times$ Solan Hara & 14.49 & 19.17 & 46.33 & 49.16 & 53.50 & 5.29 & 10.83 & 36.33 & 10.41 & 52.66 & 3.09 & 12.16 & 231.62 \\
\hline \multirow[t]{5}{*}{29} & NBGH 167 @ Check & 15.98 & 17.49 & 46.99 & 50.67 & 54.00 & 6.47 & 9.66 & 31.33 & 13.87 & 63.39 & 3.51 & 15.16 & 215.60 \\
\hline & Mean & 14.09 & 16.78 & 45.32 & 47.73 & 53.92 & 6.17 & 11.59 & 34.42 & 13.24 & 61.51 & 3.82 & 14.86 & 236.84 \\
\hline & C.V. & 5.06 & 5.43 & 3.43 & 5.23 & 3.47 & 5.74 & 7.04 & 5.41 & 5.56 & 5.74 & 5.17 & 6.54 & 5.92 \\
\hline & S.E. & 0.29 & 0.37 & 0.63 & 1.02 & 0.76 & 0.14 & 0.33 & 0.76 & 0.30 & 1.44 & 0.08 & 0.40 & 5.73 \\
\hline & C.D. $5 \%$ & 0.81 & 1.04 & 1.77 & 2.85 & 2.13 & 0.40 & 0.93 & 2.13 & 0.84 & 4.03 & 0.23 & 1.11 & 16.01 \\
\hline
\end{tabular}




\begin{tabular}{|c|c|c|c|c|c|c|c|c|c|}
\hline S. No. & Characters & Yld/Vine (g) & Yld/ha (q) & Frt Fly Inf \% & (TSS) & $\begin{array}{c}\text { Ascorbic } \\
\text { Acid } \\
\text { (mg/100g.) }\end{array}$ & $\begin{array}{c}\text { Total } \\
\text { chlorophyll } \\
\text { (mg/100g.) }\end{array}$ & $\begin{array}{c}\text { Carotenoids } \\
\text { (mg/100g.) }\end{array}$ & $\begin{array}{c}\text { Total } \\
\text { Phenol } \\
(\mathbf{m g} / \mathbf{1 0 0 g})\end{array}$ \\
\hline 1 & Pusa Ausadhi & 2097.78 & 151.80 & 20.76 & 4.92 & 49.95 & 0.59 & 1.35 & 113.84 \\
\hline 2 & Jhalri & 1716.11 & 124.18 & 24.48 & 5.60 & 57.74 & 0.78 & 0.76 & 90.42 \\
\hline 3 & Kathi No. 1 & 2015.56 & 145.85 & 18.05 & 4.88 & 76.84 & 1.12 & 1.58 & 137.49 \\
\hline 4 & PusaRasdar & 1849.38 & 133.82 & 17.28 & 4.61 & 67.97 & 0.71 & 0.97 & 126.74 \\
\hline 5 & BRBT Local & 2087.22 & 151.04 & 16.65 & 6.08 & 90.35 & 1.20 & 0.66 & 102.50 \\
\hline 6 & Konkan Tara & 1968.19 & 142.42 & 15.97 & 5.07 & 93.29 & 1.09 & 1.72 & 132.38 \\
\hline 7 & Solan Hara & 1444.93 & 104.56 & 19.78 & 4.69 & 64.08 & 0.56 & 0.91 & 92.39 \\
\hline 8 & Pusa Ausadhi $\times$ Jhalri & 2229.79 & 161.35 & 24.11 & 5.10 & 53.81 & 0.60 & 1.36 & 119.36 \\
\hline 9 & PusaAusadhi $\times$ Kathi No. 1 & 2411.04 & 174.47 & 23.89 & 4.86 & 62.38 & 0.85 & 1.47 & 127.58 \\
\hline 10 & PusaAusadhi $\times$ PusaRasdar & 2343.14 & 169.55 & 21.48 & 4.56 & 56.10 & 0.73 & 1.29 & 123.73 \\
\hline 11 & Pusa Ausadhi $\times$ BRBT Local & 2630.21 & 190.33 & 20.30 & 5.61 & 59.55 & 0.94 & 1.02 & 115.58 \\
\hline 12 & PusaAusadhi $\times$ Konkan Tara & 2398.33 & 173.55 & 20.94 & 4.97 & 67.82 & 0.77 & 1.70 & 126.68 \\
\hline 13 & PusaAusadhi $\times$ Solan Hara & 1955.83 & 141.57 & 25.78 & 5.02 & 57.10 & 0.52 & 1.20 & 110.38 \\
\hline 14 & Jhalri $\times$ Kathi No.1 & 2033.96 & 147.15 & 24.83 & 5.89 & 65.70 & 0.80 & 0.94 & 111.38 \\
\hline 15 & Jhalri $\times$ PusaRasdar & 1879.79 & 136.03 & 25.06 & 5.79 & 63.95 & 0.81 & 1.17 & 101.24 \\
\hline 16 & Jhalri $\times$ BRBT Local & 2047.29 & 148.14 & 26.58 & 6.87 & 65.18 & 1.10 & 0.80 & 100.63 \\
\hline 17 & Jhalri $\times$ Konkan Tara & 1983.92 & 143.56 & 24.19 & 5.72 & 67.60 & 0.85 & 1.15 & 115.69 \\
\hline 18 & Jhalri $\times$ Solan Hara & 1723.61 & 124.72 & 26.69 & 5.49 & 59.96 & 0.69 & 0.94 & 91.53 \\
\hline 19 & Kathi No. $1 \times$ PusaRasdar & 2223.61 & 160.90 & 22.57 & 4.98 & 74.09 & 0.91 & 1.57 & 140.97 \\
\hline 20 & Kathi No. $1 \times$ BRBT Local & 2587.85 & 187.26 & 22.59 & 5.83 & 92.06 & 1.23 & 1.46 & 98.43 \\
\hline 21 & Kathi No. $1 \times$ Konkan Tara & 2175.62 & 157.38 & 22.38 & 4.94 & 97.44 & 1.02 & 2.22 & 147.40 \\
\hline 22 & Kathi No. $1 \times$ Solan Hara & 1962.15 & 141.99 & 19.38 & 5.02 & 77.13 & 0.66 & 1.75 & 129.24 \\
\hline 23 & PusaRasdar $\times$ BRBT Local & 2110.49 & 152.69 & 21.34 & 4.81 & 83.36 & 1.00 & 1.17 & 124.32 \\
\hline 24 & PusaRasdar $\times$ Konkan Tara & 2007.64 & 145.28 & 18.91 & 5.31 & 85.38 & 0.81 & 1.77 & 132.57 \\
\hline 25 & PusaRasdar $\times$ Solan Hara & 1828.54 & 132.32 & 22.08 & 4.41 & 83.47 & 0.66 & 1.28 & 129.95 \\
\hline 26 & BRBT Local × Konkan Tara & 2552.78 & 184.72 & 17.98 & 6.18 & 107.37 & 1.32 & 1.50 & 127.50 \\
\hline 27 & BRBT Local $\times$ Solan Hara & 2025.97 & 146.60 & 22.82 & 6.47 & 91.86 & 1.05 & 1.11 & 101.92 \\
\hline 28 & Konkan Tara $\times$ Solan Hara & 1952.36 & 141.28 & 23.60 & 5.59 & 101.21 & 0.91 & 1.98 & 139.51 \\
\hline \multirow[t]{5}{*}{29} & NBGH 167 @ Check & 2073.04 & 149.20 & 25.98 & 4.49 & 67.66 & 0.77 & 0.86 & 127.21 \\
\hline & Mean & 2079.87 & 150.47 & 21.95 & 5.30 & 73.81 & 0.87 & 1.30 & 118.57 \\
\hline & C.V. & 5.30 & 6.49 & 7.01 & 3.76 & 3.40 & 10.77 & 5.08 & 3.15 \\
\hline & S.E. & 45.03 & 3.99 & 0.63 & 0.08 & 1.03 & 0.04 & 0.03 & 1.52 \\
\hline & C.D. $5 \%$ & 125.91 & 11.14 & 1.76 & 0.23 & 2.87 & 0.11 & 0.08 & 4.26 \\
\hline
\end{tabular}


Fruit weight, fruit length and fruit diameter are also the important traits for getting higher yields. BRBT Local (82.71g) followed by PusaRasdar $(65.10 \mathrm{~g})$ and Pusa Ausadhi (55.91g) were promising parents for the trait, fruit weight. Among hybrids Pusa Ausadhi X BRBT Local (91.43 g) followed by Kathi No.1 X BRBT Local (83.13g) and PusaRasdar X BRBT Local (81.11g) were promising hybrids. Similarly for fruit length BRBT Local $(16.41 \mathrm{~cm})$ followed by Jhalri $(14.19 \mathrm{~cm})$ and Pusa Ausadhi $(13.17 \mathrm{~cm})$ were desirable parents and the hybrids Pusa Ausadhi X BRBT Local $(17.92 \mathrm{~cm})$ followed by BRBT Local X Konkan Tara $(17.30 \mathrm{~cm})$ and Kathi No.1 X BRBT Local $(15.63 \mathrm{~cm})$ were most promising hybrids for this trait. Among the parental lines maximum diameter of fruit was recorded for PusaRasdar (4.89 $\mathrm{cm})$ followed by BRBT Local $(4.72 \mathrm{~cm})$ and Kathi No. $1(4.22 \mathrm{~cm})$. Among F1 hybrids the maximum fruit diameter was recorded by Pusa Rasdar X BRBT Local $(4.87 \mathrm{~cm})$ followed by Pusa Ausadhi X BRBT Local $(4.84 \mathrm{~cm})$ and thus these were the desirable genotypes. For total number of seed per fruit, Solan Hara (21.33) followed by Kathi No.1 (20.33) had maximum number of seed per fruit among genotypes while among hybrids, BRBT Local X Solan Hara (22.83) had comparatively high number of seed per fruit, followed by Kathi No.1 X BRBT Local (17.11) . Low infestation of fruit fly might increase the fruit yield. Minimum fruit fly infestation was recorded in Konkan Tara (15.97\%) followed by BRBT Local (16.65 $\%)$ among parents and promising BRBT Local X Konkan Tara (17.98 \%) followed by Pusa Rasdar X Konkan Tara (18.91 \%) for this trait. Similar types of finding were also observed in bitter gourd by Islam et al., (2014) and Singh et al., (2016)

Fruit yield is the ultimate character which shows the utility of a genotype in farmer's field. As per the present findings, the parents
Pusa Aushadhi (151.80 q/h) followed by BRBT Local (151.04 q/ha) and Kathi No.1 (145.85 q/ha) were the most promising. Among hybrids, Pusa Ausadhi X BRBT Local $(190.33 \mathrm{q} / \mathrm{ha})$ followed by Kathi No.1 X BRBT Local (187.26 q/ha) and BRBT Local $\mathrm{X}$ Konkan Tara (184.72 q/ ha) were promising. Similar trend of results were also found by Jadhav et al., 2009, Rani et al., (2014) and Kumar et al., (2017).

Among the quality traits, maximum total soluble solid (TSS) was in BRBT Local (6.08 ${ }^{0}$ Brix) followed by Jhalri (5.60 ${ }^{0}$ Brix $)$. Among hybrids Jhalri X BRBT Local $\left(6.87^{0}\right.$ Brix $)$ followed by BRBT Local X Solan Hara (6.47 ${ }^{0}$ Brix) were promising hybrid for this trait. Highest ascorbic acid content was recorded in Konkon Tara $(93.29 \mathrm{mg} / 100 \mathrm{~g})$ followed by BRBT Local (90.35 mg/100g) and Kathi No.1 (76.84 mg /100g). Out of 21 hybrids, BRBT Local X Konkon Tara (107.37) had highest ascorbic acid content followed by Kathi No.1 X Konkon Tara (97.44 mg /100g). These findings were in conformity with the findings of Thangamani and Pugalendhi (2013) for ascorbic acid content and Mallikarjunarao K. et al., (2018) for TSS. Among parental line highest total chlorophyll content was in BRBT Local $(1.20 \mathrm{mg} / 100 \mathrm{~g})$ and Kathi No.1 (1.12 mg/ 100g). The hybrid BRBT Local X Konkon Tara (1.32 mg/ 100g) and Kathi No.1 X BRBT Local (1.23) were promising hybrids for this qualitative trait. Carotenoid content was highest in Konkon Tara $(1.72 \mathrm{mg} / 100 \mathrm{~g})$ followed by Kathi No.1 $(1.58 \mathrm{mg} / 100 \mathrm{~g})$ while hybrid Kathi No.1X Konkon Tara (2.22 mg/ 100g) and Konkan Tara X Solan Hara were considered as promising hybrid for this trait. For total phenol content highest amount was recorded in Kathi No. 1 (137.49 mg/ 100g) followed by Konkan Tara (132.38 mg/ 100g) and in hybrid Kathi No.1X Konkon Tara (147.40 mg/ 100g) followed by Kathi No.1X PusaRasdar (140.97 $\mathrm{mg} / \mathrm{100 \textrm {g }}$ ) were considered as promising 
hybrid for this trait. For crop improvement programme, selection of parents is most crucial and important step which need careful attention. From the present investigation it is concluded that among the parental lines, Pusa Ausadhi were the earliest with respect to node number of $1^{\text {st }}$ male flower and node number of $1^{\text {st }}$ female flower, days taken to $1^{\text {st }}$ male flowers appearance, days taken to $1^{\text {st }}$ female flowers appearance and days to $50 \%$ flowering. While, the minimum internodal length was found with Konkan Tara. The other parameters like vine length, number of primary branches, fruit weight and fruit length were found to be best for the parent, BRBT Local. Fruit yield is the ultimate character which shows the utility of a genotype in farmer's field. As per the present findings, the parents Pusa Ausadhi, followed by BRBT Local and Kathi No.1were the most promising parental line. However, among hybrids, Pusa Ausadhi X BRBT Local followed by Kathi No.1 X BRBT Local and BRBT Local X Konkan Tara were the promising hybrid regarding fruit yield. From the quality point of view, the best promising parent were found to be BRBT Local for total soluble solid (TSS) and chlorophyll content, Konkan Tara, for carotenoid content, Kathi No. 1, for total phenol content and Konkan Tara, for ascorbic acid. Out of 21 hybrids, BRBT Local X Konkan Tara had highest ascorbic acid content followed by Kathi No.1 X Konkan Tara.

\section{References}

Agrawal, M. and Kamal, R. 2004. In vitro clonal propagation of Momordica charantia L. Indian Journal of Biotechnology, 3: 426-430.

Behera, T.K., Gaikwad, A.B, Singh, A.K. and Staub, J.E. 2008. Comparative analysis of genetic diversity in Indian bitter gourd (Momordica charantia L.) using RAPD and ISSR markers for developing crop improvement strategies. Scientia Horticulturae, 115: 209-217

Beloin, N., Gbeassor, M., Akpagana, K., Hudson, J., Koumaglo, K. and Arnason, J.T 2005.Ethnomedicinal uses of Momordica charantia (Cucurbitaceae) in Togo and relation to its phytochemistry and biological activity. Journal of Ethnopharmacoogy, 96: 4955.

Grover, J.K and Yadav, S.P 2004. Pharmacological actions and potential uses of Momordica charantia. A Review Journal of Ethnopharmacology, 93(1):123-132.

Islam, M., Mia, M.A.B., Das, M. R, Hossain, T., Ahmed, J.U. and Hossain, M.M. 2014. Sex phenology of bitter gourd (Momordica charantia L.) landraces and its relation to yield potential and fruit quality. Pak. J. Agri. Sci., 51(3): 651-658.

Jadhav, K.A., Garad, B.V., Dhumal, S. S., Kshirsagar, D. B., Patil, B. T. and Shinde K. G. 2009. Heterosis in bitter gourd. (Momordica charantia L.) Agri. Sci. Digest., 29: 7-11.

Mallikarjunarao, K., Das, A.K., Nandi, A., Baisakh, B., Sahu, G.S. and Tripathy, P. 2018. Evaluation of Parents and Hybrids for Yield and Quality characters in Bitter Gourd (Momordica charantia L.). Int.J.Curr.Microbiol. App.Sci. 7(08): 1082-1092

Mini Raj, N., Prasanna, K.P. and Peter, K.V. 1993. Bitter Gourd: Momordica spp. p. 239-246. In G. Kalloo and B.O. Bergh (eds.). Genetic Improvement of Vegetable Crops. Pergamon Press, Oxford, U.K.

Panse, V.G. and Sukhatme, P.V. 1957. Statistical Methods for Agricultural Workers. Indian Council of Agricultural Research, New Delhi. Pp. 97.

Rajeswari, K.S. 1998. Genetic studies in bitter 
gourd (Momordica charantia L.) through diallel analysis. M.Sc. (Hort.) Thesis, Tamil Nadu Agricultural University, Coimbatore.

Raman, A. and Lau, C. 1996. Anti-diabetic properties and phytochemistry of Momordica charantia L. (Cucurbitaceae). Phytomedicine 2(4): 349-362.

Rani, K.R., Ch S, Raju, and Reddy, K.R., 2014. A study on heterosisi for yield and earliness in Bitter gourd.Indian Journal of Scientific Research and Technology, 2(3): 89-97

Singh, V., Shah, K.N. and Rana, D.K 2016. Performance of different bitter gourd (Momordica charantia L.) strain for growth, yield and quality traits under Garhwal Hills. Plant Arch., 16(2): 815820.

Singh, V., Shah, K.N and Rana, D.K. 2016. Performance of different bitter gourd
(Momordica charantia L.) strain for growth, yield and quality traits under Garhwal Hills. Plant Arch., 16(2): 815820.

Sundaram, V. 2006. Studies on genetics of yield and yield components in bitter gourd (Momordica charantia L.) under salinity. Ph.D. (Hort.) Thesis, Tamil Nadu Agricultural University, Coimbatore.

Zafar, R. and Neerja, 1991. Momordica charantia-a review. Hamdard Medicine, 34: 49-61.

Zehra, S.B, Parveen, K. Hussain K, HabibMehfuza, Dar ZA and GulShaheen (2018). Per se performance of bitter gourd (Momordica charantia L.) genotypes under temperate conditions of Kashmir. International Journal of Chemical Studies, 6(2): 3158-3160

\section{How to cite this article:}

Amrita Kumari, Sangeeta Shree, Randhir Kumar, M. Haque, Chandan Kishor and Singh, V.K. 2019. Study of Per se Performance of Parents and Hybrids for Yield and Quality of Bittergourd (Momordica charantia L.). Int.J.Curr.Microbiol.App.Sci. 8(07): 1781-1789.

doi: https://doi.org/10.20546/ijcmas.2019.807.212 